\title{
Clinical outcomes of intracerebral hemorrhage in hemodialysis patients
}

Noriaki Sakamoto a, c, Eiichi Ishikawa c, Kazuyasu Aoki a, b, Yoji Uemae a, Yoji Komatsu a, c, Akira Matsumura ${ }^{\mathrm{c}}$

a Department of Neurosurgery, Hitachi General Hospital, Ibaraki

${ }^{\mathrm{b}}$ Department of Neurosurgery, Nerima Hikarigaoka Hospital, Tokyo

${ }^{\mathrm{c}}$ Department of Neurosurgery, Faculty of Medicine, University of Tsukuba, Ibaraki

Key words: Intracerebral hemorrhage, Hemodialysis, Chronic renal failure, Hypertension

Running head on left-hand page: N. Sakamoto et al.

Running title on right-hand page: Intracerebral hemorrhage in hemodialysis patients

Address reprint requests to: Eiichi Ishikawa, MD, PhD, Department of Neurosurgery, Faculty of Medicine, Graduate School of Comprehensive Human Sciences, University of Tsukuba, 1-1-1 Tennodai, Tsukuba, Ibaraki 305-8575, Japan. e-mail: e-ishikawa@md.tsukuba.ac.jp 


\section{Introduction}

Chronic renal failure (CRF) is a worldwide public health problem that is associated with a high risk of occurrence of cardiovascular events $[9,19]$. Hemodialysis (HD) is performed in more than $90 \%$ of patients in advanced stages of CRF. The number of HD patients in Japan has increased from 1624.1 per million at the end of 2000 to 2279.5 per million in 2009 . The total number of HD patients exceeded 281,996 in 2009, the highest rate since 1983 [17]. The trend is similar in other countries, the total number of HD patients in the United States increasing from 281,355 in 2000 to 415,013 in 2010 (United States Renal Data System) $[4,26]$. Several studies have indicated that the incidence of ischemic and hemorrhagic events in the intracranial region (strokes) in CRF patients is high $[1,7,8,10,12,14$ $16,18,21-23,27]$. A single-center study in Japan showed that the frequency of intracerebral hemorrhage ( $\mathrm{ICH}, 52 \%$ of 151 patients) in HD patients was higher than that of cerebral infarction (Cl, 41\%) between 1980 and 1996, while the rate of $\mathrm{ICH}(29 \%)$ between 1997 and 2002 was lower than that of $\mathrm{Cl}(68 \%)$ [23]. Intensive control of hypertension, diabetes and hyperlipidemia may have reduced the incidence of $\mathrm{ICH}$. However, the clinical status of HD patients with $\mathrm{ICH}$ remains severe, $\mathrm{ICH}$ being a common cause of death in HD patients. The incidence of death due to $\mathrm{ICH}$ is 2- to 3-fold higher than that due to $\mathrm{Cl}[8,10,12,15]$.

Here, we retrospectively investigated 5 years worth of clinical data from ICH patients treated with or without HD at our institution. We reveal the differences in the clinical courses of HD and non-HD patients and identify the risk factors for poor outcomes in ICH patients undergoing HD.

\section{Patients and Methods}

We conducted a single-center retrospective study based upon a review of medical records. 
The records of 366 consecutive patients with non-traumatic spontaneous ICH who were admitted to the neurosurgical department of Hitachi General Hospital between January 2007 and December 2011 were examined. Patients with ICH due to secondary causes, such as ruptured aneurysm, primary ischemic stroke, and arteriovenous malformation or tumor, were excluded from this study. In all cases, computed tomography (CT) scan was performed on admission. Magnetic resonance imaging (MRI) was performed on admission or after surgery for all patients with ICH except for deceased patients whose condition was rapidly deteriorating.

The following clinical information, including baseline characteristics, was collected: age, gender, location/side of the hematoma, volume of the hematoma, presence of intraventricular hemorrhage (IVH), microbleeds (MBs) on MRI scans, modified Rankin scale (mRS) scores at admission/discharge, type of surgical operation, use of antihypertensive and anti-diabetic drugs and antiplatelets/anticoagulants, primary renal disease, current history of $\mathrm{HD}$, and the day of the week on which the ICH occurred in relation to HD. The hematoma volume in each case was determined by one of the authors (N.S.) from CT scans obtained at the onset as follows: the maximum transverse diameter $\times$ the maximal antero-posterior diameter $\times$ the maximal supero-inferior diameter $\times 1 / 2$. Presence of IVH was also assessed using CT scans. Hematoma volume in the lateral ventricle was not included in the calculation of 'volume of hematoma' in this study.

The values are expressed as means \pm SD. Differences in patient data were evaluated using univariate logistic analysis as well as $\chi^{2}$ test, Fisher's test or Student t test. P values less than 0.05 were considered to indicate statistical significance. All calculations were performed using JMP 5 software (SAA Corp., USA). Multivariate logistic analysis was performed for data in which $\mathrm{p}$ values were less than 0.1 on univariate logistic analyses. Differences were considered statistically significant if the $p$ value was $<0.05$ in this analysis 
as well. In the logistic analyses, continuous variables were dichotomized in terms of their mean or median values.

\section{Results}

\section{Comparisons between HD and non-HD patients}

Table 1 shows the clinical characteristics of the patients in the study. A total of 366 patients were admitted to our hospital with a diagnosis of $\mathrm{ICH}$ during the study period. They were divided into two groups: 32 patients (9\%) with CRF who received HD (HD group) and 334 patients who did not receive HD treatment (non-HD group). All the CRF patients in this study were on HD. Surgical hematoma evacuation was performed in $25 \%$ of the HD patients and in $13.5 \%$ of the non-HD patients, while a hematoma drain was inserted into the lateral ventricle of $6 \%$ and $7.5 \%$ of HD and non-HD patients, respectively. There were no differences in patient age, gender, laterality of hematoma and surgical procedure between the two groups. The HD group had higher rates of hematomas in the basal ganglia (84\% in the HD group vs. $57 \%$ in the non-HD group, $p<0.05)$, IVH (53\% vs. 34\%, $p<0.05)$, use of antihypertensive drugs ( $72 \%$ vs. $32 \%, p<0.01)$, use of anti-diabetic drugs ( $41 \%$ vs. $20 \%, p<0.01)$ and use of antiplatelets/anticoagulants (41\% vs. $17 \%, \mathrm{p}<0.01)$. The mortality rate (mRS VI) was higher in the HD group (44\%) than in the non-HD group (21\%). There were no significant differences in the existence of cerebral MBs between the two groups, as seen on MRI ( $p>0.1$, Fisher direct test). As seen in Table 1, univariate logistic analysis showed that hematoma location, presence of IVH, use of antihypertensive drugs, use of anti-diabetic drugs, use of antiplatelets/anticoagulants, patient mortality, and the number of patients with $\mathrm{mRS} \geq 5$ were significantly different between HD and non-HD groups. Similar results were also shown using the $\chi^{2}$ test or Fisher's test (data not shown). Multivariate logistic analysis showed that 
hematoma location, use of antihypertensive drugs, use of antiplatelets/anticoagulants, and number of patients with $\mathrm{mRS} \geq 5$ were different between the two groups (Table 1).

Analysis of patients who had died (mRS VI) in both groups indicated significant differences in the number of hematomas in the basal ganglia ( $86 \%$ vs. $45 \%, p<0.01$ ), the use of antihypertensive drugs ( $50 \%$ vs. $21 \%$ : $p<0.01)$ and the use of antiplatelets/anticoagulants ( $36 \%$ vs. $12 \%, p<0.05$ ). There were no significant differences in the use of anti-diabetic drugs and incidence of IVH between the two groups.

\section{Analyses of HD patients}

Next, detailed data from the 32 patients on HD was analyzed. The causes of CRF were diabetic nephropathy (44\%), glomerulonephritis (19\%), cystic kidney disease (3\%), sclerosis (3\%), renal carcinoma (3\%) and unknown etiology (28\%). On admission, 13 patients (41\%) received antiplatelets/anticoagulants for maintenance of shunt patency (31.2\%), history of previous angina pectoris (2 cases), or history of previous cerebral infarction (1 case).

The condition of 24 of the patients (75\%) was rated as being serious (mRS V) at the time of hospitalization, and antihypertensive drugs were administered to 23 of these patients. Mean systolic blood pressure values on admission in patients with and without antihypertensive drugs before admission were $190.0+/-43.2$ and $166.3+/-53.1 \mathrm{mmHg}$ $(p>0.05$, Student $t$ test), respectively. Hematoma drain placement in the lateral ventricle was performed in 2 patients, both of who survived, while surgical hematoma evacuation was performed for 6 patients who survived and 2 patients who had a poor outcome (mRS Vl). The final outcomes were mRS VI (44\%) and V (25\%). As shown in Figure 1, most patients' conditions did not improve throughout hospitalization. We analyzed the risk factors for poor outcomes (mRS VI). As shown in Table 2, univariate logistic analysis demonstrated significant differences between the patients who did and did not survive in terms of GCS 
scores on admission, mRS scores on admission, hematoma volume, presence of IVH, and the use of antihypertensive drugs. Multivariate logistic analysis, however, showed no significant prognostic factor, indicating that the results of the analysis should only be used as an advisory, since the analyses were performed in a small sized sample. Older patients tended to have higher mortality rates than younger patients, although the difference between the two groups was not significant. There was no difference in systolic blood pressure between poor outcome patients and other HD patients $(179.8+/-59.8 \mathrm{mmHg}(\mathrm{n}=13)$ vs. 185.0+/- $35.0(n=16), p>0.1$, Student $t$ test $)$, or in HD duration between poor outcome patients and other HD patients $(7.1+/-5.4$ years $(n=13)$ vs. $7.7+/-4.3(n=15), p>0.1$, Student $t$ test).

As shown in Figure 2, no significant difference was observed between mortality (mRS VI) due to ICH on dialysis days (10 cases, 37\%) and non-dialysis days (17 cases, 63\%) in 27 cases with data regarding the timing of the $\mathrm{ICH}(\mathrm{p}>0.05$, Fisher's method). In these cases, $26 \%$ of the $\mathrm{ICH}$ occurred before the HD procedure on an HD day, which was a higher rate than during or after the HD procedure (11\%) on the dialysis day.

\section{Discussion}

On admission, the condition of ICH patients on HD in this study was frequently classified as being serious. The prognostic factors associated with mortality were GCS on admission $<8$, hematoma volume $>50 \mathrm{ml}$, the presence of $\mathrm{IVH}$, and lack of antihypertensive drug usage. In previous papers, the overall 30-day mortality rate in $\mathrm{ICH}$ patients with advanced CRF has been shown to be $30-83 \%[7,16]$. In one of the studies, the prognostic factors independently associated with mortality were as follows: GCS scores, old age, systolic blood pressure, ICH volume $\geq 30 \mathrm{ml}$, presence of $\mathrm{IVH}$, and high serum glucose [7]. In another 
paper, additional factors indicating poor prognosis were level of consciousness on admission, the size and shape of the hematoma, prothrombin time, and fibrin degradation product level [14]. In general, cerebral MBs detected on T2-weighted MRI scans are closely related to ICH $[11,13,20,24]$, although our results could not corroborate this since our data included only $\mathrm{ICH}$ patients. A previous paper showed that $35 \%$ of CRF patients had MBs. Factors associated with MBs were male sex, old age, hypertension and worsening of CRF, although MBs did not show a correlation with the duration of HD treatment [25].

In our study, antihypertensive drugs were used more frequently by CRF patients than non-CRF patients. CRF patients frequently have renal hypertension, which may cause ICH. The odds ratio for $\mathrm{ICH}$ was 2.44 in the $\mathrm{CRF}$ group when compared to the non-CRF group [22]. However, the use of antiplatelets/anticoagulants did not significantly predict poorer outcomes. Therefore, patients should strictly control their hypertension and should not hesitate to take antiplatelets/anticoagulants as they do not pose a risk for $\mathrm{ICH}$. A limitation of the present study is that the results were obtained from retrospective and non-controlled data. However, various analyses, including univariate and multivariate logistic regression analyses showed similar results. Hence, we believe that these results are generally reliable.

As shown in Figure 2, ICH frequently occurred on a non-dialysis day. HD in CRF patients is usually scheduled three times a week, and some reports show that the occurrence of disease is related to the day of HD. For example, sudden cardiac death events occur more often on Mondays and Wednesdays [2]. The occurrence rates of acute myocardial infarction, heart failure and stroke are high when the interval between HD treatments is two days [5] This suggests that medical practitioners should be careful to screen HD patients for ICH on non-dialysis days.

In our study, mortality in ICH patients on HD was associated with lack of antihypertensive drug usage. Most patients who do not use antihypertensive drugs are 
patients with absent or mild hypertension, although there may be some cases of drug withdrawal in patients with severe hypertension. Interestingly, previous studies regarding risk factors for poor outcomes in non-traumatic $\mathrm{ICH}$ also did not contain a history of hypertension $[3,6]$. For instance, one of these studies suggests that the factors independently associated with 30-day mortality are GCS scores, age $\geq 80$ years, infratentorial origin of $\mathrm{ICH}$, ICH volume and the presence of IVH [6]. In this previous study, the incidence of hypertension in the poor outcome group (43\%) was lower than that in the other ICH group (73\%) [6], similar to the present results. We speculate that severe $\mathrm{ICH}$ depends upon factors other than hypertension (such as existence of microaneurysms). Hence, the occurrence of severe $\mathrm{ICH}$ in both HD and non-HD patients should be considered independently of hypertension.

In conclusion, multivariate logistic analysis showed that hematoma location, use of antihypertensive drugs, use of antiplatelets/anticoagulants, and patient outcome were significantly different between HD and non-HD groups. On the other hand, use of antihypertensive drugs or antiplatelets/anticoagulants did not worsen the outcome of the $\mathrm{ICH}$ patients on HD. Thus, in HD patients, the possibility of severe ICH should be considered regardless of the use of antihypertensive drugs or antiplatelets/anticoagulants. In this study, $\mathrm{ICH}$ frequently occurred before the HD procedure on an HD day or on an interval day.

\section{Conflict of Interest statement}

All authors declare no conflicts of interest. 


\section{References}

1. Anavekar NS, McMurray JJ, Velazquez EJ, Solomon SD, Kober L, Rouleau JL, White HD, Nordlander R, Maggioni A, Dickstein K, Zelenkofske S, Leimberger JD, Califf RM, Pfeffer MA. Relation between renal dysfunction and cardiovascular outcomes after myocardial infarction. N Engl J Med. 2004;351:1285-95

2. Bleyer AJ, Russell GB, Satko SG. Sudden and cardiac death rates in hemodialysis patients. Kidney Int. 1999;55:1553-9

3. Cheung RT, Zou LY. Use of the original, modified, or new intracerebral hemorrhage score to predict mortality and morbidity after intracerebral hemorrhage. Stroke. 2003;34:1717-22.

4. Collins AJ, Foley RN, Herzog C, Chavers B, Gilbertson D, Herzog C, Ishani A, Johansen K, Kasiske B, Kutner N, Liu J, St Peter W, Ding S, Guo H, Kats A, Lamb K, Li S, Li S, Roberts T, Skeans M, Snyder J, Solid C, Thompson B, Weinhandl E, Xiong H, Yusuf A, Zaun D, Arko C, Chen SC, Daniels F, Ebben J, Frazier E, Hanzlik C, Johnson R, Sheets D, Wang X, Forrest B, Constantini E, Everson S, Eggers P, Agodoa L. US Renal Data System 2012 Annual Data Report. Am J Kidney Dis. 2013;61(1 Suppl 1):A7

5. Foley RN, Gilbertson DT, Murray T, Collins AJ. Long interdialytic interval and mortality among patients receiving hemodialysis. N Engl J Med. 2011;365:1099-107.

6. Hemphill JC 3rd, Bonovich DC, Besmertis L, Manley GT, Johnston SC. The ICH score: a simple, reliable grading scale for intracerebral hemorrhage. Stroke. 2001;32:891-7.

7. Huang BR, Liao CC, Huang WH, Hsu YH, Hsu JC, Yen HC, Lin CL. Prognostic factors of spontaneous intracerebral hemorrhage in hemodialysis patients and predictors of 30-day mortality. Intern Med J. 2008;38:568-74. 
8. Iseki K, Fukiyama K; Okawa Dialysis Study (OKIDS) Group. Clinical demographics and long-term prognosis after stroke in patients on chronic hemodialysis. The Okinawa Dialysis Study (OKIDS) Group. Nephrol Dial Transplant. 2000;15:1808-13

9. Iseki K, Fukiyama K. Predictors of stroke in patients receiving chronic hemodialysis. Kidney Int. 1996;50:1672-5.

10. Iseki K, Kinjo K, Kimura Y, Osawa A, Fukiyama K. Evidence for high risk of cerebral hemorrhage in chronic dialysis patients. Kidney Int. 1993;44:1086-90

11. Kato H, Izumiyama M, Izumiyama K, Takahashi A, Itoyama Y. Silent cerebral microbleeds on T2*-weighted MRI: correlation with stroke subtype, stroke recurrence, and leukoaraiosis. Stroke. 2002;33:1536-40

12. Kawamura M, Fijimoto S, Hisanaga S, Yamamoto Y, Eto T. Incidence, outcome, and risk factors of cerebrovascular events in patients undergoing maintenance hemodialysis. Am J Kidney Dis. 1998;31:991-6

13. Kinoshita T, Okudera T, Tamura H, Ogawa T, Hatazawa J. Assessment of lacunar hemorrhage associated with hypertensive stroke by echo-planar gradient-echo T2*-weighted MRI. Stroke. 2000;31:1646-50

14. Miyahara $\mathrm{K}$, Murata $\mathrm{H}, \mathrm{Abe} \mathrm{H}$. Predictors of intracranial hematoma enlargement in patients undergoing hemodialysis. Neurol Med Chir (Tokyo). 2007;47:47-51

15. Molshatzki N, Orion D, Tsabari R, Schwammenthal Y, Merzeliak O, Toashi M, Tanne D. Chronic kidney disease in patients with acute intracerebral hemorrhage: association with large hematoma volume and poor outcome. Cerebrovasc Dis. 2011;31:271-7.

16. Murakami M, Hamasaki T, Kimura S, Maruyama D, Kakita K. Clinical features and management of intracranial hemorrhage in patients undergoing maintenance dialysis therapy. Neurol Med Chir (Tokyo). 2004;44:225-32 
17. Nakai S, Iseki K, Itami N, Ogata S, Kazama JJ, Kimata N, Shigematsu T, Shinoda T, Shoji T, Suzuki K, Taniguchi M, Tsuchida K, Nakamoto H, Nishi H, Hashimoto S, Hasegawa T, Hanafusa N, Hamano T, Fujii N, Masakane I, Marubayashi S, Morita O, Yamagata K, Wakai K, Wada A, Watanabe Y, Tsubakihara Y. Overview of regular dialysis treatment in Japan (as of 31 December 2009). Ther Apher Dial. 2012;16:11-53.

18. Nakayama K, Tannir NM, Liu P, Wathen JK, Cheng YC, Champlin RE, Ueno NT. Natural history of metastatic renal cell carcinoma in patients who underwent consultation for allogeneic hematopoietic stem cell transplantation. Biol Blood Marrow Transplant. 2007;13:975-85

19. Ninomiya T, Kiyohara $Y$, Kubo M, Tanizaki Y, Doi Y, Okubo K, Wakugawa Y, Hata J, Oishi Y, Shikata K, Yonemoto K, Hirakata H, lida M. Chronic kidney disease and cardiovascular disease in a general Japanese population: the Hisayama Study. Kidney Int. 2005;68:228-36.

20. Offenbacher H, Fazekas F, Schmidt R, Koch M, Fazekas G, Kapeller P. MR of cerebral abnormalities concomitant with primary intracerebral hematomas. AJNR Am J Neuroradiol. 1996;17:573-8

21. Ovbiagele B. Chronic kidney disease and risk of death during hospitalization for stroke. J Neurol Sci. 2011;301:46-50.

22. Thrift AG, Evans RG, Donnan GA. Hypertension and the risk of intracerebral hemorrhage: special considerations in patients with renal disease. Nephrol Dial Transplant. 1999;14:2291-2

23. Toyoda K, Fujii K, Fujimi S, Kumai Y, Tsuchimochi H, Ibayashi S, lida M. Stroke in patients on maintenance hemodialysis: a 22-year single-center study. Am J Kidney Dis. 2005;45:1058-66 
24. Tsushima Y, Tanizaki Y, Aoki J, Endo K. MR detection of microhemorrhages in neurologically healthy adults. Neuroradiology. 2002;44:31-6

25. Watanabe A. Cerebral microbleeds and intracerebral hemorrhages in patients on maintenance hemodialysis. J Stroke Cerebrovasc Dis. 2007;16:30-3.

26. Xue JL, Ma JZ, Louis TA, Collins AJ. Forecast of the number of patients with end-stage renal disease in the United States to the year 2010. J Am Soc Nephrol. 2001;12:2753-8.

27. Yahalom G, Schwartz R, Schwammenthal Y, Merzeliak O, Toashi M, Orion D, Sela BA, Tanne D. Chronic kidney disease and clinical outcome in patients with acute stroke. Stroke. 2009;40:1296-303.

\section{Figure legends}

Figure 1. Modified Rankin Scale scores in intracerebral hemorrhage (ICH) patients on admission and on discharge

Figure 2 Relationship between $\mathrm{ICH}$ occurrence and the timing of the hemodialysis (HD) procedure. Black and white bars indicate the frequencies of living and deceased patients, respectively. 
Fig. 1

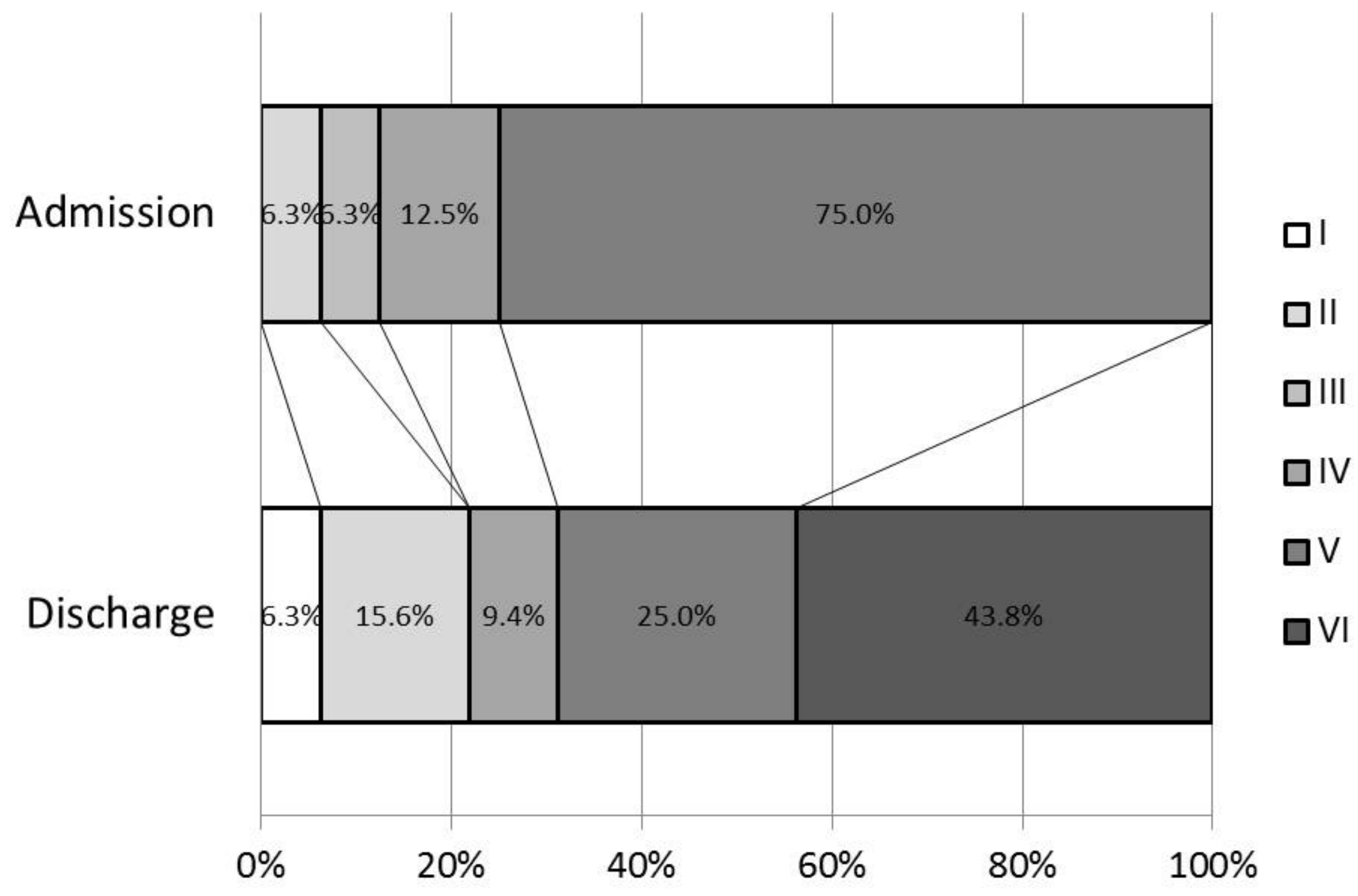




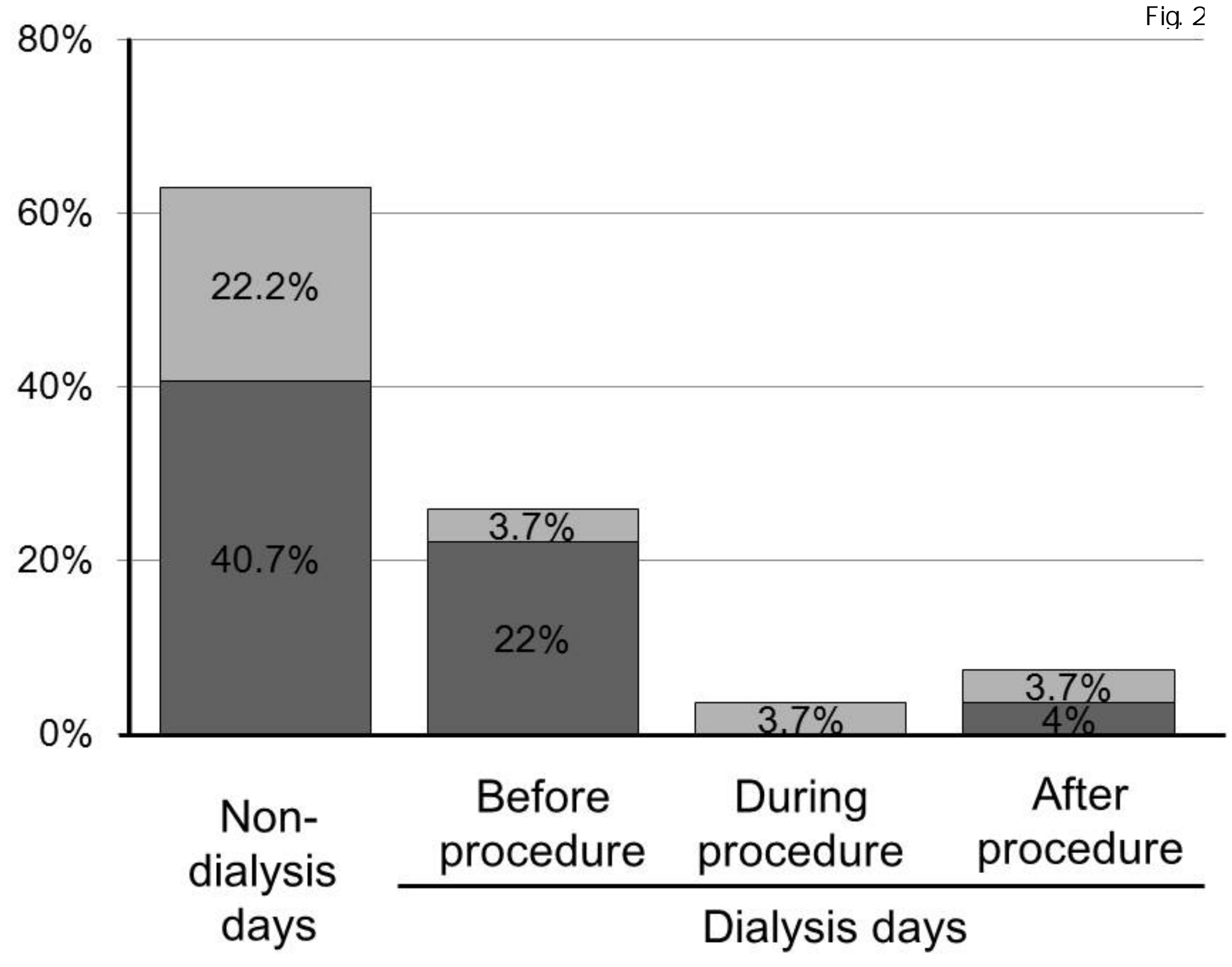


Table 1: Characteristics of ICH patients $(n=366)$

\begin{tabular}{|c|c|c|c|c|c|c|c|c|}
\hline & \multirow{2}{*}{\multicolumn{2}{|c|}{$\begin{array}{c}\mathrm{HD} \\
(\mathrm{n}=32)\end{array}$}} & \multirow{2}{*}{\multicolumn{2}{|c|}{$\begin{array}{c}\text { Non-HD } \\
(n=334)\end{array}$}} & \multicolumn{4}{|c|}{ Logistic analysis (p value, Exp) } \\
\hline & & & & & \multicolumn{2}{|c|}{ Univariate } & \multicolumn{2}{|c|}{ Multivariate } \\
\hline \multicolumn{9}{|c|}{ Background } \\
\hline Median age (years) & \multicolumn{2}{|c|}{$64(28-84)$} & \multicolumn{2}{|c|}{$69(8-95)$} & \multicolumn{4}{|c|}{$(>0.1$, Student $t)$} \\
\hline Age ( $\leq 65$ years) & 16 & $50 \%$ & 134 & $40 \%$ & $\underline{0.2801}$ & $(1.493)$ & $=$ & $=$ \\
\hline Gender (Female) & 16 & $50 \%$ & 135 & $40 \%$ & 0.2952 & (1.474) & $=$ & $=$ \\
\hline Use of $\underline{\mathrm{AHD}}(\mathrm{Y})$ & 23 & $72 \%$ & 108 & $32 \%$ & 0.0001 & $\underline{5.348}$ & 0.0009 & 4.934 \\
\hline Use of $\underline{A D D}(Y)$ & 13 & $41 \%$ & 66 & $20 \%$ & 0.0080 & $\underline{2.778}$ & $\underline{0.1880}$ & $(1.846)$ \\
\hline Use of $\underline{A P / A C}(Y)$ & 13 & $41 \%$ & 58 & $17 \%$ & $\underline{0.0023}$ & $\underline{3.256}$ & $\underline{0.0068}$ & $\underline{3.299}$ \\
\hline One druq / Two drugs & \multicolumn{2}{|c|}{$9 / 4$} & \multicolumn{2}{|c|}{$45 / 13$} & & & & \\
\hline \multicolumn{9}{|c|}{ After ICH } \\
\hline Location (BG) & 27 & $84 \%$ & 192 & $57 \%$ & $\underline{0.0055}$ & 3.994 & 0.0087 & $\underline{4.130}$ \\
\hline $\begin{array}{c}\text { BG (putamen, thalamus)/ Lobar (subcortical) } \\
\text { / Others (cerebellum, brainstem) }\end{array}$ & \multicolumn{2}{|c|}{$27 / 3 / 2$} & \multicolumn{2}{|c|}{$192 / 70 / 72$} & & & & \\
\hline Laterality (Left sided) & 18 & $58 \%$ & 166 & $56 \%$ & 0.4800 & $(1.300)$ & $=$ & $=$ \\
\hline Presence of IVH & 17 & $53 \%$ & 114 & $34 \%$ & 0.0357 & $\underline{2.187}$ & 0.7968 & $\underline{(1.127)}$ \\
\hline Operation (Y) & 10 & $31 \%$ & 70 & $21 \%$ & $\underline{0.2128}$ & (1.654) & $=$ & $=$ \\
\hline $\begin{array}{c}\text { Surgical hematoma evacuation / Hematoma } \\
\text { drain placement in the lateral ventricle }\end{array}$ & \multicolumn{2}{|c|}{$\underline{8 / 2}$} & \multicolumn{2}{|c|}{$45 / 25$} & & & & \\
\hline \multicolumn{9}{|c|}{ Patient Outcome } \\
\hline Outcome (mRS) (Vl) & 14 & $44 \%$ & 71 & $21 \%$ & $\underline{0.0054}$ & 2.881 & $\underline{0.3150}$ & $\underline{(1.741)}$ \\
\hline$(\mathrm{V}+\mathrm{VI})$ & 22 & $69 \%$ & 122 & $37 \%$ & 0.0008 & 3.823 & 0.0093 & 4.338 \\
\hline I/II/IIIIIV/V/VI & $2 / 5 / c$ & $13 / 8 / 14$ & 51/92/2 & 151/71 & & & & \\
\hline
\end{tabular}

ADD, anti-diabetic drugs; AHD, anti-hypertensive drugs; AP/AC, antiplatelets or anticoagulants; BG, Basal

ganglia; HD, hemodialysis; ICH, intracerebral hemorrhage; IVH, intraventricular hemorrhage; mRS, modified

Rankin scale; $\underline{Y, \text { yes }}$ 
Table 2: Characteristics of ICH patients with HD (n=32)

\begin{tabular}{|c|c|c|c|c|c|c|}
\hline & \multirow{2}{*}{$\begin{array}{l}\text { Dead } \\
(n=14)\end{array}$} & \multirow{2}{*}{$\begin{array}{l}\text { Alive } \\
(\mathrm{n}=18)\end{array}$} & \multicolumn{4}{|c|}{ Logistic analysis ( $p$ value, Exp) } \\
\hline & & & \multicolumn{2}{|c|}{$\underline{\text { Univariate }}$} & \multicolumn{2}{|l|}{ Multivariate } \\
\hline \multicolumn{7}{|c|}{ Background } \\
\hline Age (years) & $70.3+/-10.2$ & $62.1+/-12.7$ & \multicolumn{4}{|c|}{$(0.0591$, Student $t)$} \\
\hline Age $>65$ years & $64 \%$ & $39 \%$ & $\underline{0.1590}$ & $(2.825)$ & & \\
\hline$\underline{\text { Gender (Female) }}$ & $57 \%$ & $44 \%$ & $\underline{0.4773}$ & (1.667) & & \\
\hline$\underline{\mathrm{HD} \text { duration until } \mathrm{ICH} \text { (years) }}$ & $7.1+1-5.4$ & $\underline{7.7+1-4.3}$ & \multicolumn{4}{|c|}{$(>0.1$, Student $t)$} \\
\hline$\underline{\text { HD duration }<7.5 \text { years }}$ & $\underline{62 \%}$ & $\underline{47 \%}$ & $\underline{0.4331}$ & $\underline{1.828}$ & & \\
\hline Use of AHD (N) & $50 \%$ & $11 \%$ & $\underline{0.0240}$ & $\underline{8.000}$ & $\underline{0.9864}$ & - \\
\hline$\underline{\text { Use of ADD (N) }}$ & $\underline{64 \%}$ & $\underline{56 \%}$ & $\underline{0.6185}$ & $\underline{(1.441)}$ & & \\
\hline Use of APIAC (N) & $\underline{64 \%}$ & $\underline{56 \%}$ & $\underline{0.6185}$ & $(1.441)$ & & \\
\hline \multicolumn{7}{|c|}{ After ICH } \\
\hline GCS on admission (median) & $\underline{4}$ & $\underline{13.5}$ & & $01, M W$ & & \\
\hline$\underline{\operatorname{GCS}<8(Y)}$ & $\underline{93 \%}$ & $\underline{17 \%}$ & $\underline{0.0006}$ & $\underline{65.00}$ & $\underline{0.9480}$ & - \\
\hline$\underline{\mathrm{mRS}}$ on admission (median) & $\underline{5}$ & $\underline{5}$ & \multicolumn{4}{|c|}{$\underline{(0.0356, \mathrm{MWU})}$} \\
\hline$\underline{\mathrm{mRS}}=5$ & $\underline{93 \%}$ & $\underline{61 \%}$ & $\underline{0.0649}$ & $\underline{8.273}$ & $\underline{0.9999}$ & \\
\hline$\underline{\mathrm{sBP}(\mathrm{mmHg})}$ & $\underline{179.8+/-59.8}$ & $\underline{185.0+1-35.0}$ & \multicolumn{4}{|c|}{$(>0.1$, Student $t)$} \\
\hline$\underline{\mathrm{sBP}<180}$ & $\underline{38 \%}$ & $\underline{38 \%}$ & $\underline{0.9577}$ & $\underline{(1.042)}$ & & \\
\hline$\underline{\mathrm{ICH}}$ location (BG) & $86 \%$ & $78 \%$ & $\underline{0.5709}$ & $(1.714)$ & & \\
\hline$\underline{\mathrm{ICH}}$ laterality (non-left sided) & $54 \%$ & $33 \%$ & $\underline{0.2578}$ & $\underline{(2.333)}$ & & \\
\hline$\underline{\mathrm{ICH}}$ volume & $74.3+/-16.4$ & $24.0+/-6.2$ & \multicolumn{4}{|c|}{$(0.0027$, Student $t)$} \\
\hline Volume >50 ml (Y) & $58 \%$ & $17 \%$ & $\underline{0.0240}$ & $\underline{6.993}$ & $\underline{0.9612}$ & - \\
\hline Presence of IVH (Y) & $79 \%$ & $33 \%$ & $\underline{0.0152}$ & $\underline{7.353}$ & $\underline{0.9856}$ & - \\
\hline Operation (N) & $\underline{86 \%}$ & $\underline{56 \%}$ & $\underline{0.0810}$ & $\underline{4.808}$ & $\underline{0.9496}$ & - \\
\hline \multicolumn{7}{|c|}{$\underline{\text { Patient Outcome }}$} \\
\hline$\underline{\text { GOS (median) }}$ & $\underline{5}$ & $\underline{3}$ & \multicolumn{4}{|c|}{ (0.0001, MWU) } \\
\hline$\underline{\mathrm{mRS} \text { (median) }}$ & $\underline{6}$ & $\underline{4}$ & \multicolumn{4}{|c|}{$(0.0001, M W U)$} \\
\hline
\end{tabular}


$\underline{A D D}$, anti-diabetic drugs; AHD, anti-hypertensive drugs; AP/AC, anti-platelets or anti-coagulants; BG, basal ganglia; GCS, Glasgow Come Scale; GOS, Glasgow Outcome Scale; ICH, intracerebral hemorrhage; IVH, intraventricular hemorrhage; mRS, modified Rankin scale; MWU, Mann-Whitney U test; N, no; sBP, Systolic Blood pressure on admission; $Y$, yes. 